\title{
Original
}

\section{Brotes de diarrea e intoxicaciones transmitidas por alimentos en Costa Rica, 2005}

\author{
Hilda Ma. Bolaños-Acuña, María Teresa Acuña-Calvo, Francisco Duarte-Martínez, \\ Wagner Salazar-Castro, Gletty Oropeza-Barrios, Luz Marina Sánchez-Salazar, \\ Elena Campos-Chacón
}

Centro Nacional de Referencia en Bacteriología,

Instituto Costarricense de Investigación y Enseñanza en Nutrición y Salud (INCIENSA)

Abreviaturas: CNRB, Centro Nacional de Referencia en Bacteriología, INCIENSA; ELISA, ensayo inmunoabsorbente ligado a enzima; ETA, enfermedad transmitida por alimentos; ETEC, Escherichia coli enterotoxigénica;VETA, Sistema de Vigilancia de Enfermedades Transmitidas por Alimentos. Correspondencia: Hilda Ma. Bolaños Acuña, Centro Nacional de Referencia en Bacteriología, Instituto Costarricense de Investigación y Enseñanza en Nutrición y Salud (INCIENSA)

E-mail: hbolanos@inciensa.sa.cr

ISSN 0001-6002/2007/49/4/205-209 Acta Médica Costarricense, (O2007 Colegio de Médicos y Cirujanos

\section{Resumen}

Justificación y objetivo: En la actualidad, las enfermedades de transmisión alimentaria, incluyendo las diarreas e intoxicaciones, constituyen uno de los problemas de salud pública de mayor importancia a nivel mundial, ya que ocasionan alta morbilidad y mortalidad, generan grandes costos a los servicios de salud, pérdidas económicas, demandas y pérdida de confianza de los consumidores. El objetivo del trabajo es analizar la información disponible en el Centro Nacional de Referencia en Bacteriología del INCIENSA (CNRB), de los brotes de diarrea e intoxicaciones alimentarias ocurridos en 2005, en los que se refirieron muestras clínicas o de alimentos, para identificar los agentes y vehículos de infección asociados.

Materiales y métodos: Se realizó el análisis descriptivo de la información epidemiológica y de laboratorio disponible en el CNRB en relación con los brotes de diarrea e intoxicaciones alimentarias ocurridos en 2005.

Resultados: Durante el período el CNRB documentó 23 brotes de diarrea e intoxicaciones alimentarias, para un total aproximado de 819 afectados, en su mayoría procedentes de la gran área metropolitana. Trece de los brotes fueron intrafamiliares (diez de ellos debidos a especies y serotipos de Shigella); sin embargo, el mayor número de enfermos se presentó en los brotes comunitarios y los que se produjeron en poblaciones cautivas, asociados a rotavirus, Escherichia coli enterotoxigénica y Clostridium perfringens productor de toxina A, con 518, 138 y 54 enfermos, respectivamente.

Conclusión: Estos hallazgos evidencian deficiencias en la cadena de transporte, conservación y manipulación de los alimentos, así como en la higiene personal, y pueden ser de utilidad para la implementación de intervenciones inmediatas.

Descriptores: diarrea, enfermedad transmitida por alimentos, intoxicación alimentaria, Shigella spp, Salmonella spp, C. perfringens toxina A, E. coli enterotoxigénica, Staphylococcus aureus, rotavirus

Key words: diarrhea, alimentary intoxications

Recibido: 7 de febrero de 2007

Aceptado: 5 de junio de 2007

Según estimaciones de la Organización Mundial de la Salud (OMS), la incidencia anual de diarrea en el mundo es de 1500 millones de casos, con una mortalidad anual de 3 millones de niños menores de 5 años. Se estima además que el $70 \%$ de las diarreas se originan por la ingestión de alimentos contaminados con microbios y sus toxinas, en cantidades suficientes para afectar la salud del consumidor, por lo que se reconocen como enfermedades transmitidas por 
alimentos (ETA). ${ }^{1,2}$ Entre los agentes etiológicos de diarrea se encuentran algunos que producen infecciones y que cuando alcanzan la luz intestinal pueden multiplicarse y producir toxinas o invadir la pared intestinal y desde ahí alcanzar otros aparatos o sistemas, como es el caso de Salmonella spp., Shigella spp., Campylobacter spp., rotavirus, mientras que las intoxicaciones alimentarias (como las producidas por Staphylococcus aureus, Bacillus cereus), se producen cuando las toxinas de bacterias o mohos ya están presentes en el alimento ingerido. ${ }^{3}$

En la actualidad, estas enfermedades constituyen uno de los problemas de salud pública de mayor importancia a nivel mundial, ya que ocasionan alta morbilidad y mortalidad, generan grandes costos a los servicios de salud, pérdidas económicas y de la confianza de los consumidores así como demandas. ${ }^{1}$ El problema se ha incrementado en los últimos años debido a la globalización, la producción centralizada de alimentos y a los cambios en los hábitos alimentarios. Por lo anterior, organismos internacionales como la Organización Panamericana de la Salud (OPS)/OMS han recomendado el establecimiento de sistemas de vigilancia de estas enfermedades que permitan detectar oportunamente la aparición de brotes, conocer la magnitud del problema y diseñar estrategias de prevención y control de las mismas. ${ }^{2,3}$ En Costa Rica aún no se cuenta con un sistema de vigilancia de las enfermedades de transmisión alimentaria (VETA), por lo que no se dispone de información sistematizada sobre el problema. El Centro Nacional de Referencia en Bacteriología del INCIENSA (CNRB), desde hace 15 años ha brindado apoyo al sistema de vigilancia epidemiológica y a los laboratorios de la red nacional en la investigación de brotes de diarrea que se presentan en el país. En este documento se resume la información recopilada por el CNRB para el período 2005, con el fin de que pueda ser de utilidad para la implementación de un sistema VETA y para la toma de decisiones en el corto, mediano y largo plazo.

\section{Materiales y métodos}

Para cada uno de los brotes estudiados por el CNRB entre enero y diciembre de 2005 , se completó un formulario con datos básicos (fecha de inicio, lugar de ocurrencia, ubicación geográfica, número estimado de afectados, patógeno identificado en los casos, alimento identificado como vehículo de infección), a partir de los datos brindados por los encargados locales de la investigación o de las boletas de confirmación diagnóstica referidas por los microbiólogos de los laboratorios de la red nacional.

En el CNRB las muestras de heces y cepas referidas se procesaron por métodos previamente descritos para el aislamiento e identificación, pruebas serológicas y moleculares para la detección de enteropatógenos. ${ }^{4-6}$ El diagnóstico de rotavirus se realizó con el kit ELISA
Ridascreen ${ }^{\mathrm{TM}}$ (Bio-pharma GMBH, Darmstadt, Germany) y la determinación de toxinas de Staphylococcus aureus y Clostridium perfringens mediante pruebas de aglutinación pasiva reversa (SET-RPLA y PET-RPLA, Denka Seiken Co. Tokio, Japan). Además, se emplearon las tinciones tricrómica modificada y Ziehl Neelsen para realizar la búsqueda de parásitos intestinales. Los alimentos considerados de mayor riesgo de acuerdo con la información clínico-epidemiológica disponible se analizaron según las metodologías convencionales, dando prioridad a la búsqueda del patógeno identificado en los casos clínicos asociados al brote. ${ }^{7}$ Una vez depurada, la información resumida de cada brote se digitó y analizó utilizando estadística descriptiva en una base de datos Excel.

\section{Resultados}

Durante 2005 el CNRB dio apoyo al sistema de vigilancia epidemiológica y a los laboratorios de la red nacional en la investigación de 23 brotes, que afectaron a un total aproximado de 819 personas (Cuadro 1). Trece (57\%) de estos brotes fueron intra-familiares, diez de ellos debidos a especies y serotipos de Shigella (con un promedio de dos afectados por brote). Estos brotes por Shigella se detectaron a partir del proceso de confirmación diagnóstica/tipificación, originándose la alerta desde el laboratorio. Seis brotes (26\%) se produjeron en poblaciones cautivas (que incluyen servicios de alimentación de fábricas, un servicio de pediatría de un hospital y dos centros penitenciarios) y cuatro (17\%) se catalogaron como brotes comunitarios. Los brotes con mayor impacto por el número de personas enfermas fueron los comunitarios debidos a rotavirus (aproximadamente 518 afectados) y los que se dieron en poblaciones cautivas (con aproximadamente 192 afectados), asociados a Escherichia coli enterotoxigénica y Clostridium perfringens productor de toxina A. Entre los brotes por rotavirus, el ocurrido en Chacarita, Puntarenas, a inicios de 2005, fue el que tuvo el mayor número de enfermos (447) que solicitaron atención médica en los servicios de salud.

No se registraron defunciones asociadas a los brotes estudiados, a diferencia de lo ocurrido en otros años.

Muestras de alimentos sospechosos se recibieron en el CNRB únicamente en siete de los 23 brotes, en cinco de los cuales fue posible demostrar la presencia del agente identificado en los casos; por ejemplo, entre de los brotes por Salmonella, uno se debió a la serovariedad Newport (brote a) y se relacionó con el consumo de alimentos en una soda de una comunidad y en el otro por Salmonella Weltevreden (brote b), no fue posible documentar el alimento sospechoso.

En dos de los brotes en que los afectados resultaron positivos por Clostridium perfringens toxina A se determinó la presencia de este agente en pierna de cerdo (brote c) y en 
Cuadro 1. Brotes de diarrea e intoxicaciones alimentarias por tipo, según Patógeno Costa Rica, 2005

\begin{tabular}{|c|c|c|c|c|c|}
\hline \multirow{2}{*}{$\begin{array}{l}P \quad a \quad t \quad o ́ \\
\text { identificado }\end{array}$} & \multicolumn{3}{|c|}{ Número de brotes según tipo } & \multirow{2}{*}{$\begin{array}{l}\text { Total de brotes } \\
\text { con patógeno } \\
\text { identificado }\end{array}$} & \multirow{2}{*}{$\mathrm{N}^{\circ}$ afectados } \\
\hline & Intrafamiliar & $\begin{array}{c}\text { Poblaciones } \\
\text { cautivas* }^{*}\end{array}$ & Comunitario & & \\
\hline $\begin{array}{l}\text { Especies y serotipos de } \\
\text { Shigella }\end{array}$ & 10 & 0 & 0 & 10 & $\geq 24$ \\
\hline S. sonnei & 4 & 0 & 0 & 4 & $\geq 8$ \\
\hline S. flexneri $2 a$ & 2 & 0 & 0 & 2 & $\geq 4$ \\
\hline S. flexneri $3 a$ & 3 & 0 & 0 & 3 & $\geq 10$ \\
\hline S. flexneri prov, 101 & 1 & 0 & 0 & 1 & $\geq 2$ \\
\hline $\begin{array}{l}\text { Serovariedades de } \\
\text { Salmonella }\end{array}$ & 1 & 0 & 1 & 2 & $\geq 48$ \\
\hline S. Newport & 0 & 0 & $1^{(\mathrm{a})}$ & 1 & $\geq 45$ \\
\hline S. Weltevreden & $1^{\text {(b) }}$ & 0 & 0 & 1 & $\geq 3$ \\
\hline $\begin{array}{l}\text { Escherichia coli } \\
\text { enterotoxigénica (ETEC) }\end{array}$ & 0 & 2 & 0 & 2 & $\geq 138$ \\
\hline $\begin{array}{l}\text { Clostridium perfringens } \\
\text { toxina A positivo }\end{array}$ & $1^{(\mathrm{c})}$ & $2^{(d)}$ & 0 & 3 & $\geq 68$ \\
\hline Staphylococcus aureus & $1^{(\mathrm{g})}$ & $1^{(\mathrm{g})}$ & $1^{(\mathrm{g})}$ & 3 & $\geq 23$ \\
\hline Rotavirus & 0 & 1 & 2 & 3 & $\geq 518$ \\
\hline Total de brotes & 13 & 6 & 4 & 23 & \\
\hline $\mathrm{N}^{\circ}$ aprox. afectados & $\geq 46$ & $\geq 200$ & $\geq 573$ & & $\geq 819$ \\
\hline
\end{tabular}

* Incluye dos fábricas, un servicio de pediatría de un hospital, dos centros penitenciarios.

ensalada (brote d), mientras que en las tres intoxicaciones por Staphylococcus aureus se logró determinar la toxina del agente en quesos de fabricación artesanal (brotes g).

La mayoría de los brotes ocurrieron en las regiones Central Sur (62\%), Central Norte y Central Este. Durante el período no se documentaron brotes en las regiones Huetar Norte, Chorotega y Atlántica.

Los brotes se presentaron durante todos los meses del año, con excepción de octubre (Figura 1); sin embargo, los de mayor impacto por el número de afectados se produjeron en enero y febrero.

\section{Discusión}

Los brotes incluidos en este reporte constituyen sin duda la "punta del iceberg" de lo que sucede en el país, por lo que se requiere fortalecer la coordinación entre los servicios de atención, los epidemiólogos, laboratorios, regulación sanitaria, entre otros, con el fin de mejorar la vigilancia de estas enfermedades y por ende su prevención y control. En vista de que el país aún no cuenta con un VETA y de que los brotes son eventos de notificación obligatoria, ${ }^{8}$ se recomienda implementar una boleta para la notificación de los brotes de

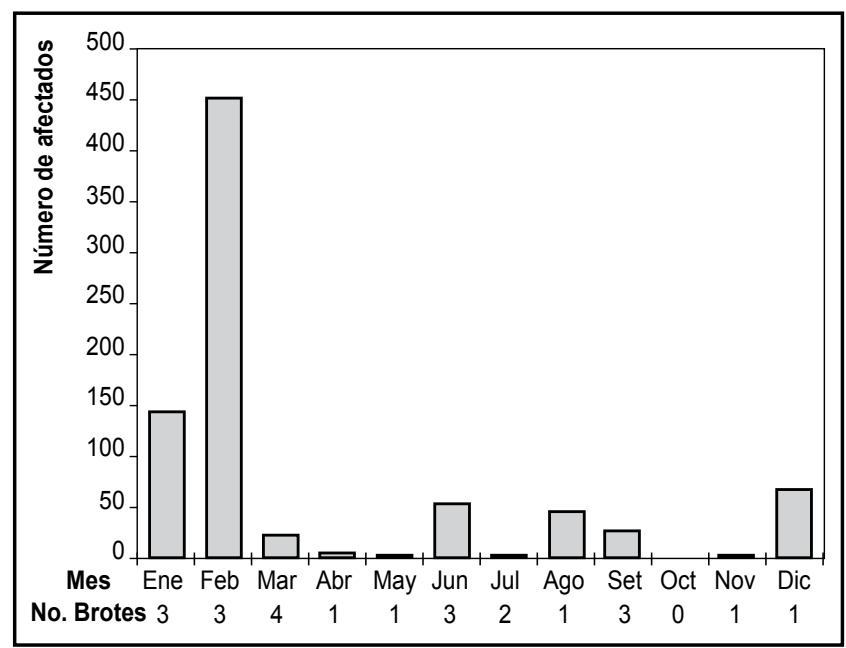

Figura 1. Número de brotes y personas afectadas por mes, Costa Rica, 2005

diarrea e intoxicaciones alimentarias al departamento de vigilancia epidemiológica del Ministerio de Salud.

La mayor parte de los brotes estudiados fueron intrafamiliares, debidos a Shigella; principalmente sonnei y flexneri (serotipos 2a y 3a), lo que probablemente está relacionado con la baja dosis infecciosa de este agente, situación que favorece su transmisión intradomiciliar. ${ }^{9}$ 
Durante el período no se documentaron brotes de Shigella de gran magnitud asociados al consumo de agua contaminada (situación que se ha presentado previamente en el país).

Los brotes que tuvieron mayor impacto por el número de personas enfermas fueron los comunitarios y los que se produjeron en poblaciones cautivas. Los brotes por C. perfringens, comúnmente se relacionan con deficiencias en el almacenamiento, manipulación y mantenimiento de los alimentos una vez cocinados, en especial carnes y sus derivados, así como legumbres, lo que concuerda con el hallazgo de una pierna de cerdo como vehículo de infección en uno de los brotes por el agente y de ensalada en otro. ${ }^{9,10}$ Estudios realizados en Costa Rica han demostrado la contaminación por C. Perfringens en carnes procesadas, molidas y embutidos muestreados en expendios y de carnes cocinadas en restaurantes. ${ }^{10}$

En el caso de los brotes por ETEC, el CNRB no recibió muestras de alimentos para análisis; sin embargo, estos con frecuencia se relacionan con el consumo de agua contaminada o de vegetales irrigados con agua no potable y recientemente son comunes los informes de brotes por ETEC en turistas a bordo de cruceros y en hoteles $9,11,12$.

A diferencia de lo ocurrido en otros años cuando se documentaron brotes de gran magnitud asociados a Salmonella Enteritidis y S. Typhimurium (CNRB, INCIENSA), en 2005 no se detectaron brotes asociados a estos agentes. A pesar de que en ninguno de los brotes por Salmonella se recolectaron alimentos para análisis, vale la pena mencionar que S. Newport se encuentra entre las diez serovariedades de Salmonella más comúnmente confirmadas en muestras clínicas, tanto en Costa Rica, como en otros países miembros de la Red Global Salm Surv. ${ }^{13}$ Esta serovariedad se ha confirmado en el país en agua para consumo humano y hay reportes de brotes asociados al consumo de carne poco cocinada, hamburguesas, queso no pasteurizado, alfalfa, entre otros. ${ }^{14}$ Por su parte, Salmonella Weltevreden es una serovariedad común en Asia (Tailandia, Vietnam), donde se ha aislado de pescados, mariscos, albahaca y hongos secos. ${ }^{13,15}$

En el caso de rotavirus, el brote ocurrido en Chacarita, Puntarenas, en febrero de 2005, concordó con un incremento de la vigilancia del agente en el país, a raíz de la alerta de brotes y defunciones por el agente en El Salvador y Nicaragua. Algunas de las muestras de este brote positivas por rotavirus se refirieron al Centro para el Control de Enfermedades (CDC, Atlanta, GA), donde se confirmaron cepas de rotavirus pertenecientes a los genotipos G1P8, G4P8 y G9P8. En este sentido, es importante destacar la necesidad de disponer del diagnóstico en los laboratorios del país, ya que este agente se asocia comúnmente a casos aislados y brotes de diarrea, que afectan principalmente a menores de edad. Por otra parte, es indispensable implementar la vigilancia de los serotipos de rotavirus circulantes, lo que podría ser de utilidad ante la eventual entrada de una vacuna al país. ${ }^{17}$

Las tres intoxicaciones por Staphylococcus aureus se asociaron al consumo de queso fresco de fabricación casera. Estudios colaborativos realizados entre Ministerio de Salud, INCIENSA e INISA han demostrado que la ingesta de queso fresco representa un riesgo a la salud del consumidor, ya que contiene niveles de Staphylococcus aureus, Listeria monocytogenes y coliformes fecales que sobrepasan los límites aceptables según las normativas.

El conocimiento de los agentes asociados a brotes y los vehículos de infección debe servir de insumo para las actividades de promoción de la salud dirigidas a la población en general, para la capacitación de manipuladores de alimentos y de los funcionarios encargados de evaluar los servicios de alimentación, entre otros. Sin embargo, la investigación epidemiológica se deberealizaroportunamente, de manera que sea posible obtener las muestras, tanto clínicas como de los alimentos sospechosos, con la brevedad posible, para facilitar la recuperación de los agentes involucrados y el establecimiento de medidas de prevención y control específicas.

Agradecimientos. Este trabajo se realizó en el marco del convenio CCSS-INCIENSA 2005. Se agradece a los funcionarios de los servicios de salud e instituciones que se indican a continuación, el haber aportado muestras, cepas o información incluidos en este estudio: Dirección Regional Central Norte y Central Sur-Min. Salud, Dirección Regional Central Norte-CCSS, Cl. Alajuelita, Cl. Aserrí, Cl. Barva, Cl. Jorge Volio, Cl. Chacarita, Cl. Santa Bárbara, Coopesalud, Coopesana, Servisalud, H. Golfito, H. Guápiles, H. Max Terán, H. Monseñor Sanabria, H. San Francisco de Asís, H. San Vicente de Paúl, Áreas Rectoras de Salud de Alajuelita, Grecia, San Antonio de Desamparados - San Francisco de Dos Ríos, Moravia y Escazú-ASEMECO-CCSS, Escuela Bello Horizonte de Escazú, Centro de Atención Integral Dr. Gerardo Rodríguez

\section{Abstract}

Justification and objective: Food borne diseases including diarrhea and food poisoning represent one of the major public health problems worldwide, since they cause high morbidity and mortality, generate great health service and economic losses, law suits and consumer distrust. This study analyze information available in the Bacteriology National Reference Center from INCIENSA, regarding diarrhea and food poisoning outbreaks that took place in the year 2005, where clinical and/or food samples were sent to INCIENSA in order to identify the agents and vehicles associated with the infections. 
Materials and methods: A descriptive analysis of the laboratory and epidemiological data concerning the 2005 foodborne intoxications and diarrheal outbreaks was performed.

Results: During 2005, CNRB documented a total of 23 diarrheal outbreaks and food poisonings for an approximate total of 819 cases, mainly from the Great Metropolitan Area. Thirteen of the outbreaks were intra-familiar (ten of them due to different Shigella species and serotypes); however, the largest number of cases was found in community outbreaks and in captive populations, associated with rotavirus, enterotoxigenic Escherichia coli and toxin A producer Clostridium perfringens, with 518, 138 and 54 cases respectively.

Conclusion: These findings suggest deficiencies in the transportation, conservation and manipulation food chain, including personal hygiene and could be useful for the implementation of immediate interventions.

\section{Referencias}

1. Helms M, Simonsen J, Molbak K. Foodborne bacterial infection and hospitalization. Clin Infect Dis. 2006;42:498-506.

2. Vigilancia epidemiológica de enfermedades transmitidas por los alimentos y sistemas de alerta en materia de inocuidad de los alimentos. [Monografía en Internet]. En: Segundo Foro Mundial FAO/OMS de Autoridades de Reglamentación sobre Inocuidad de los Alimentos. Bangkok, Tailandia: FAO/OMS. 12-14 de octubre de 2004. [Accedido: 1 de diciembre de 2006]. Disponible en: www.fao.org/docrep/ meeting/008/j3200s.htm

3. Guía de sistemas de vigilancia de las enfermedades transmitidas por alimentos (VETA) y la investigación de brotes. [Monografía en Internet]. Buenos Aires, Argentina: INPPAZ - OPS - OMS, 2001. Disponible en: http://epi.minsal.cl/epi/html/software/guias/VETA/ homepage.htm

4. Farmer JJ, Kelly T. Enterobacteriaceae. In: Balows A, Hausler NJ, Herrmann KL, Isenberg HD, Shadomy HJ, editors. Manual of clinical microbiology. Washington, D.C.: American Society for Microbiology Press: 1991. p 360-383.
5. Kelly MT, Hickman-Brenner FW, Farmer JJ. Vibrio. In: Balows A Hausler NJ, Herrmann KL, Isenberg HD, Shadomy HJ, eds. Manual of clinical microbiology. Washington, D.C.: American Society for Microbiology Press: 1991. p. 384-395.

6. Olsvik O, Strockbine A. PCR detection of heat-stable, heat-labile and shiga like toxin genes in Escherichia coli. In: Persing D, ed. Diagnostic molecular microbiology: Principles and applications. Washington, D.C.: American Society for Microbiology Press: 1993. p. 271-281.

7. Food and Drug Administration. Center for Food Safety and Applied Nutrition. Bacteriological analytical manual on line. [Monografía en Internet]. Maryland, USA: FDA; 2001. [Accedido: 25 de octubre de 2006]. Disponible en: http://www.cfsan.fda.gov/omm/microbio. $\mathrm{html}$

8. Decreto de notificación obligatoria de enfermedades No. 30945-S. La Gaceta, 27 de enero de 2003.

9. Chin J. El control de las enfermedades transmisibles. $17^{\mathrm{a}}$ ed. Washington, DC: OPS, 2001.

10. Rodríguez E, Gamboa MM, Vargas P. Clostridium perfringens en carnes crudas y cocidas y su relación con el ambiente en Costa Rica. Arch Latinom Nutr. 2002; 52:155-159.

11. Qadri F, Svennerholm AM, Faruque AS, Bradley R. Enterotoxigenic Escherichia coli in developing countries: Epidemiology, microbiology, clinical features, treatment and prevention. Clin Microbiol Rev. 2005; 18:465-483.

12. Daniels NA. Enterotoxigenic Escherichia coli: traveler's diarrhea comes home. Clin Infect Dis. 2006; 42:335-336.

13. Global Salm Surv country databank. [Base de datos en Internet]. Ginebra, Suiza: World Health Organization Global Salm-Surv (WHO GSS); 2001-2005. [Accedido: 1 de diciembre de 2006]. Disponible en: www.who.int/salmsurv/activities/en/

14. Outbreak of multidrug-resistant Salmonella Newport - United States, January-April 2002. MMWR. 2002; 51:545-548.

15. Bangtrakulnonth A, Pornreongwong S, Pulsrikarn C, Sawanpanyalert P, Hendriksen RS, Lo Fo Wong D, et al. Salmonella serovars from humans and other sources in Thailand, 1993-2002 Emerg Infect Dis. 2004; 10:131-136.

16. Demczuk W, Ahmed R, Woodward D, Clark C, King L, Dore K et al. Laboratory surveillance data for enteric pathogens in Canadá: Annual Summary 2001. Enteric Disease Program, National Microbiology Laboratory, The Canadian Science Centre for Human and Animal Health. Manitoba, Canada. 2001.

17. Hoshino Y, Kapikian AZ. Rotavirus serotypes: classification and importance in epidemiology, immunity, and vaccine development. J Health Popul Nutr. 2000; 18:5-14.

\begin{tabular}{c}
\hline $\begin{array}{c}\text { Asociación Costarricense } \\
\text { de Endocrinología }\end{array}$ \\
Actividad: \\
VI Seminario de Diabetes Mellitus \\
Fecha: 8 y 9 de noviembre de 2007 \\
Créditos de emc: 10 \\
Local: Colegio de Médicos y Cirujanos \\
Informes de la actividad \\
$232-3433$ ext 133
\end{tabular}

